\title{
Incorporating Risk into Control Design for Emergency Operation of Turbo-Fan Engines
}

\author{
Avishai Weiss ${ }^{1}$ and Ilya Kolmanovsky ${ }^{2}$ \\ The University of Michigan, Ann Arbor, Michigan, 48109-2140, USA \\ Walter Merrill ${ }^{3}$ \\ Scientific Monitoring, Inc, Scottsdale, Arizona 85258, USA
}

\begin{abstract}
The paper describes a risk function based approach to constrained turbo-fan engine control to facilitate aircraft emergency maneuvering during take-off or landing. With this approach, the pontwise-in-time state and control constraints limiting the engine thrust response can be intelligently relaxed depending on the individual engine condition monitoring. The proposed control functionality uses the barrier Lyapunov functions, the predictive reference governor and functional limiters. The approach is illustrated using simulations on a linearized and on a fully nonlinear NASA C-MAPSS40k turbo-fan engine model. The capability and flexibility of the design approach to provide fast thrust response while handling multiple constraints, that may be time-varying and dynamically changing, by coordinating multiple engine actuators is demonstrated.
\end{abstract}

\section{Nomenclature}

$\begin{array}{ll}V B V & =\text { Variable Bleed Valve } \\ \mathrm{VSV} & =\text { Variable Stator Vane } \\ W_{f} & =\text { Fueling rate } \\ F_{n} & =\text { Engine net thrust } \\ N_{c} & =\text { Core speed } \\ N_{f} & =\text { Fan speed } \\ \mathrm{LPM} & =\text { Low Pressure Compressor stall/surge margin } \\ \mathrm{HPM} & =\text { High Pressure Compressor stall/surge margin } \\ \mathrm{EPR} & =\text { Engine Pressure Ratio } \\ T_{50} & =\text { Exhaust temperature } \\ P s_{3} & =\text { Combustor inlet pressure } \\ \mathrm{SG} & =\text { Speed Gradient } \\ \mathrm{x} & =\text { Vector of state variables } \\ \mathrm{u} & =\text { Vector of control variables } \\ (\cdot)_{d} & =\text { Set-points }\end{array}$

\section{Introduction}

$\mathrm{T}$ urbo-fan engine thrust response is limited by numerous pointwise-in-time state and control constraints ${ }^{[2,9,12,14]}$. These constraints protect the engine components from unwanted operating conditions, including compressor stall or surge, excessive fan and core speeds that can cause fan blade rupture or turbine disk burst, high exhaust and

\footnotetext{
${ }^{1}$ Doctoral student, Department of Aerospace Engineering, The University of Michigan, 1320 Beal Avenue, Ann Arbor, MI 48109, AIAA member.

${ }^{2}$ Professor, Department of Aerospace Engineering, The University of Michigan, 1320 Beal Avenue, Ann Arbor, MI 48109, USA, AIAA member.

${ }^{3}$ Vice President and General Manager, Scientific Monitoring, Inc., 8777 E. Via de Ventura Dr., Suite 201

Scottsdale, Arizona 85258, U. S. A, AIAA Senior Member.
} 
metal temperatures that can cause the turbine blade meltdown, insufficient fuel to match combustor inlet pressure (lean combustor blowout), and others. Existing approaches to handle constraints are based primarily on limiting minimum and maximum quantity of fuel. The limits are set conservatively to protect the engine components for the worst case variability caused by aging, operating conditions, manufacturing tolerances and service variability across the aircraft fleet ${ }^{[9,14]}$.

The improved transient response capability of the turbo fan engines (Figure 1) has been identified as an enabler for handling a variety of emergency maneuvers by aircraft ${ }^{[1,12]}$. These include avoiding another aircraft incursion on the runway during the take-off, and the flight control through the differential thrust by two (or more) engines in case of primary aerodynamic surface (e.g., rudder) failures when the aircraft is landing. In these emergency situations, both faster thrust response and increased level of thrust response are desirable $e^{[12,14]}$.

The opportunities to improve engine transient response capability in emergency situations have been discussed in the prior literature ${ }^{[12]}$. We note here one potential opportunity that includes the use of advanced nonlinear and predictive control techniques to systematically handle constraints, and optimally coordinate Fueling rate, Variable Bleed Valve (VBV) and Variable Stator Vane (VSV) actuators of the engine. The aggressive use of the actuators to provide fast thrust response and satisfy various constraints at the engine level requires, however, that the control constraints due to the limits of these actuator themselves be carefully handled in combination with the primary engine constraints. Along these lines, an adaptive engine controller can be designed and augmented with a switching functionality to handle hard constraints ${ }^{[17]}$.

Besides changes in the engine control system, another opportunity to improve engine transient response lies in dynamically configuring and intelligently relaxing the constraints based on the on-board estimate of health/condition of a particular engine ${ }^{[14]}$. Central to this approach is the use of risk functions which predict the risk associated with constraint violation in individual components of the engine, and relate this risk to parameters in the constraints. In emergency situations, the constraints can be relaxed subject to maintaining acceptable level of risk, and the controller can be made to respond to adjusted constraints. This approach is exemplified schematically in Figure 2.

In previous work ${ }^{[14]}$, risk functions were defined as static functions of current values of engine variables to provide a quantitative measure of the potential engine failure/damage risk caused by engine variables violating the specified limits. A fuzzy inference of risk level was also proposed, with fuzzy subsets / degrees of membership linked with risk functions. It is discussed ${ }^{[14]}$, in reference to handling Surge Margin, that the control gain can be made inversely proportional to the risk so that "high bandwidth (high gain) thrust response is maintained in the low risk area" and a switch is made to a "high gain controller that quickly moves SM [Surge Margin] away from the stall line when in the high risk area".

To realize the intended controller behavior ${ }^{[14]}$, here we rely on the use of Speed-Gradient (SG) nonlinear control techniques ${ }^{[4,10,11]}$. The SG control is based on first selecting a goal function and then generating the control signal to dynamically minimize this goal function based on the gradient of the speed of change of the goal function with respect to the control signal. With this approach, the closed-loop stability is verified a posteriori using semi-definite Lyapunov function techniques. By augmenting the goal function with a risk function (which essentially acts as a penalty/barrier function for output variables approaching the limits), the control action becomes driven both by the gradient of the speed of the original goal function and by that of the risk function. The overall effect is that the system variables are pushed away from the limits when the risk function value starts to grow. This approach bears resemblance to potential field methods used for obstacle avoidance in robotics applications, where an artificial increase in potential energy around the obstacle is created to generate a force pushing the robot away from the obstacle $^{[7]}$.

We have also found, given the multitude and different nature of the constraints in the engine, that the controller design can be improved by augmenting the SG controller by the predictive reference governor. The reference governor ${ }^{[5,6]}$ modifies the set-point to the nominal controller when it becomes necessary to enforce the constraints such as the ones induces by maintaining the risk function value below a prescribed threshold. The reference governor is introduced since the actuator ability to handle the engine constraints is itself limited by actuator limits, and these limits can become active given a high gain control action induced by the risk functions. Thus the reference governor predictively maintains the actuators within the range where they retain their authority Another motivation for the use of the reference governor is that several of the constraint outputs exhibit nonminimum phase characteristics with respect to certain inputs, hampering the use of high gain SG controller to handle these constraints. While the reference governor alone is, in principle, sufficient to enforce all the constraints, our simulation results suggest that faster transient response can be obtained if the reference governor is combined with the SG controller. Other advantages of SG control include low computing time and effort and the reliance on feedback, whenever permitted by system properties, sampling rates and sensing capability, rather than precise constraint modeling to enforce the constraints. 
Functional limiters are non-predictive feed-forward constraint handling schemes that can be used, for instance, to limit the fuel quantity. Compared to SG control, which is primarily a feedback strategy, the functional limiters require an accurate model of the constraints. At the same time, functional limiters can avoid chattering that may emerge in the discrete-time implementation of SG controllers if the update rate is slow. The bounds used by the functional limiters can be adjusted based on the risk functions.

The paper is organized as follows. In Section II we review the concepts of the risk functions. In Section III we discuss the main ingredients our control approach - the Speed-Gradient control, the functional limiters and the reference governor. In Section IV we illustrate the controller design and responses using a linearized simulation model. In Section V we discuss the simulation results based on the controller implementation on the NASA CMAPSS40k model ${ }^{[13]}$. Finally, concluding remarks are made in Section VI

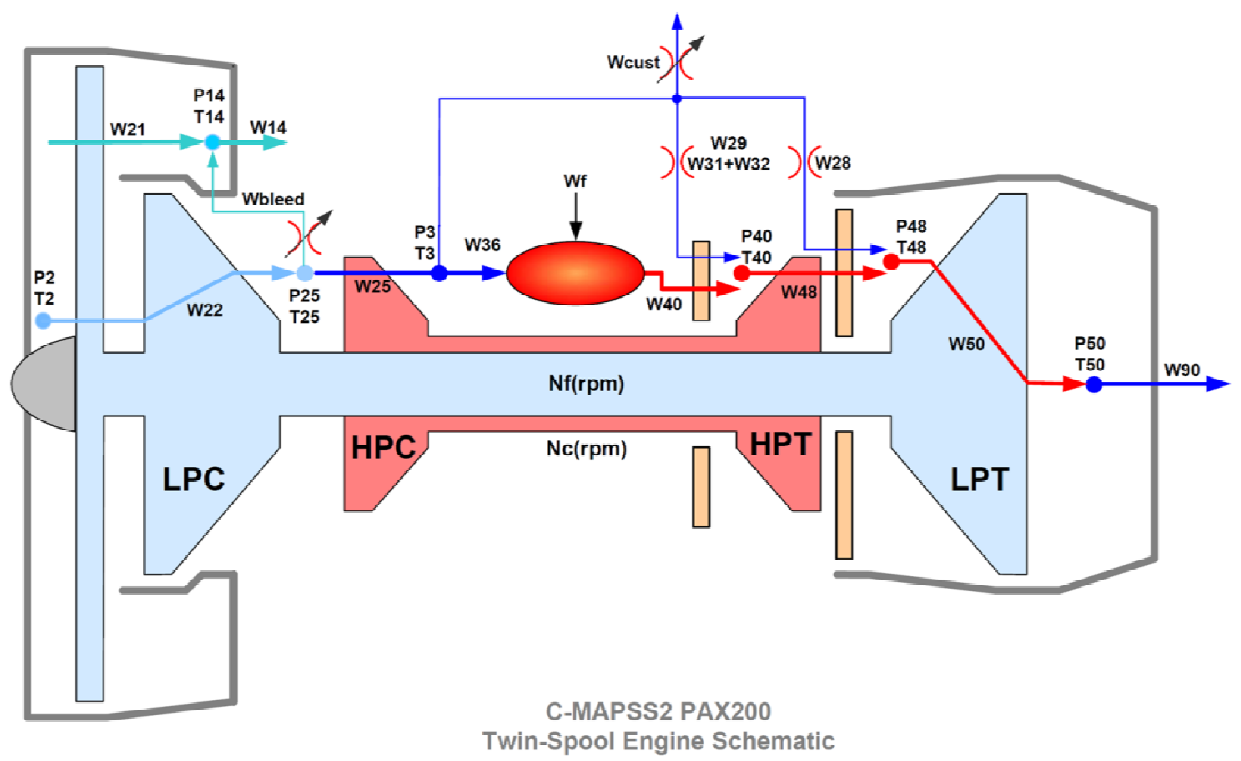
Figure 1. A schematic diagram of the twin-spool turbo-fan aircraft engine. See $e^{[2,3,9,13]}$
for component and variable definitions.

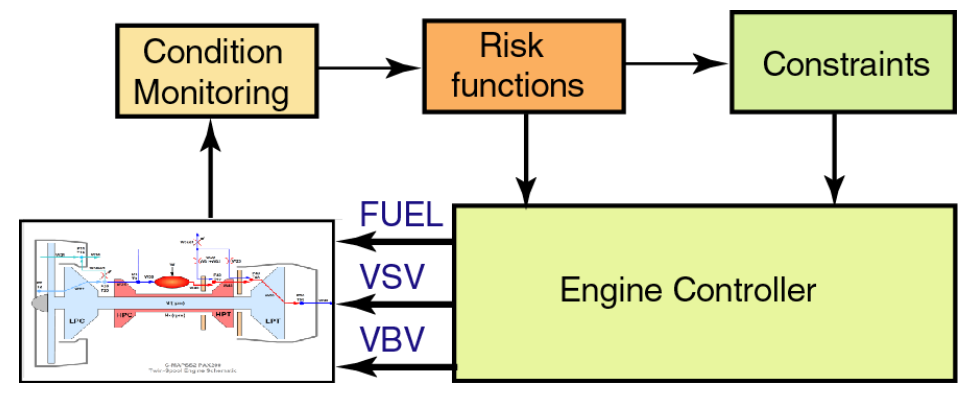

Figure 2. The risk-based framework for turbo-fan engine control during aircraft emergency maneuvering.

\section{Risk Functions}

The risk functions were introduced by Merrill ${ }^{[14]}$ as a nonlinear measure of closeness of an engine variable to constraint violation. A risk function is non-negative and monotonically increases as a given variable approaches the prescribed limit. 


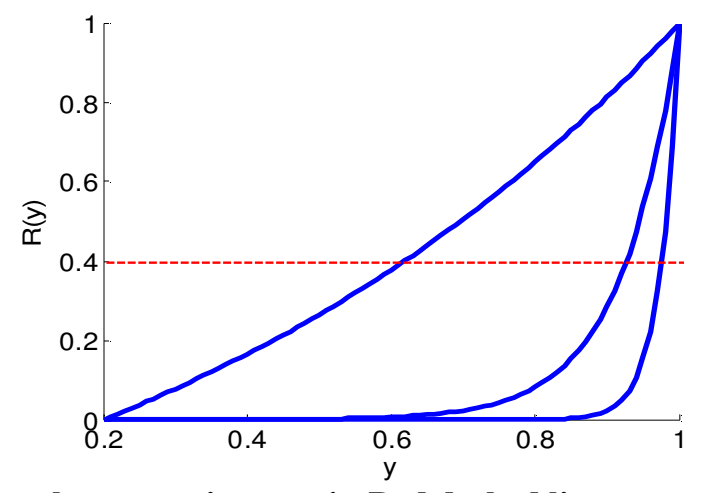

Figure 3. Risk functions for the constraint $y \leq 1$. Red dashed line suggests acceptable level of risk.

An example of a risk function corresponding to the constraint $y \leq y_{\max }$ is given by

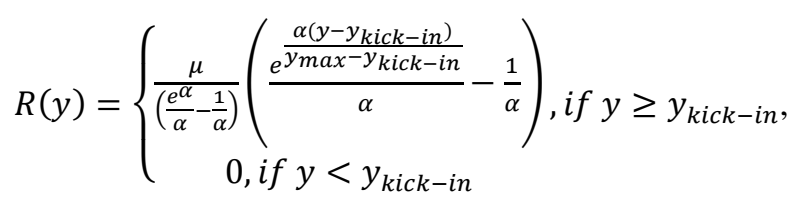

where the shape parameters of the risk function are $\alpha, \mu$ and $y_{k i c k-i n}$. This risk function satisfies the property that $R\left(y_{\max }\right)=\mu$ and $R(y)=0$ if $y<y_{\text {kick-in }}$. Figure 3 illustrates this risk function, $R(y)$, for the constraint $y \leq$ $y_{\max }=1, \mu=1, y_{\text {kick-in }}=0.2$ and varying $\alpha=1,10,30$. Merrill ${ }^{[14]}$ advocates the use of risk functions that grow infinitely large as the distance between the variable and the bound decreases, i.e.,

$$
R(y)=\frac{\mu}{1-e^{-\alpha\left(y_{\max }-y\right)}} \text { or } R(y)=\frac{\mu}{\left(y_{\max }-y\right)^{2}}
$$

A related risk function, that resembles the barrier function of Tee et. al. ${ }^{[16]}$, can be defined as follows:

$$
R(y)=\frac{1}{2} \log \frac{\mu}{\left(y_{\max }-y\right)^{2}} .
$$

In all of these cases, the risk function is a nonlinear function of the distance between the variable and its bound, that grows large once the variable approaches the bound. From the perspective of theory, the use of barrier functions that grow unbounded is preferred, however, the associated growth in the effective control gain can cause chattering and be problematic for discrete-time implementation.

We note that the engine health monitoring in Figure 2 determines the parameters of the risk function. We also note that if $\bar{R}$ represents an acceptable level of risk then the constraint $R(y) \leq \bar{R}$ induces an equivalent constraint on the variable itself, i.e., $y \leq y_{\max }(\bar{R})$. In other words, the risk functions provide nonlinear mapping between acceptable levels of risk and bounds on the variable itself.

\section{Constrained Control Framework}

The basic framework to incorporate the risk functions into the control design is based on the Lyapunov conrol technique which is referred to as the Speed-Gradient control ${ }^{[4,10,11]}$. Additionally, the risk functions can be incorporated into the functional limiters and the reference governor that handle constraints that the Speed Gradient control is not able to handle. 


\section{A. Speed-Gradient Control Based on Risk Functions}

The Speed-Gradient (SG) control is based on first selecting a goal function and then generating the control signal to dynamically minimize this goal function based on the gradient of the time rate of change of the goal function with respect to the control signal. With this approach, the closed-loop stability is verified a posteriori using Lyapunov function techniques.

Consider a model for engine dynamics in the form affine in the control inputs, i.e.,

$$
\begin{gathered}
\dot{x}=f(x)+\sum_{k=1}^{m} g_{k}(x) u_{k}, \\
y=h(x),
\end{gathered}
$$

where $y=\left(y_{0}, y_{1}, y_{2}, \cdots, y_{l}\right), y_{0}$ is the output for which the tracking set-point is prescribed, $y_{1}, y_{2}, \cdots, y_{l}$ are the outputs on which constraints are prescribed, $x$ is the state and $u_{k}$ denotes the k-th control input, $k=1, \cdots m$.

Suppose that $Q(x)=Q\left(y_{0}(x)\right)$ is a positive semi-definite function goal function for the nominal (unconstrained) closed-loop system and suppose that the performance objectives can be specified in terms of minimizing this function. The dynamic minimization of the values of $Q(x(t+\Delta t))$, augmented by an appropriate control penalty, is achieved by the following Speed-Gradient control law ${ }^{[4,10,11]}$

$$
u_{k}(t)=u_{d, k}-\Pi_{\mathrm{k}} \mathrm{L}_{\mathrm{g}_{\mathrm{k}}} \mathrm{Q}(\mathrm{x}(\mathrm{t})), \quad \Pi_{\mathrm{k}}>0, k=1, \cdots, m,
$$

where $u_{d, k}$ is the feedforward (desired control value in steady-state) and the term $\mathrm{L}_{\mathrm{g}_{\mathrm{k}}} \mathrm{Q}=\frac{\partial \mathrm{Q}}{\partial \mathrm{x}} \mathrm{g}_{\mathrm{k}}$ is the "speedgradient", i.e., the derivative of the time rate of change of the goal function, $\dot{Q}(x(t))$, with respect to the k-th control signal, $u_{k}$. To further enhance the SG controller by capability to compensate for constant additive disiturbances, this controller can be augmented by an integral action:

$$
u_{k}(t)=u_{d, k}-\Pi_{\mathrm{k}} \mathrm{L}_{\mathrm{g}_{\mathrm{k}}} \mathrm{Q}(\mathrm{x}(\mathrm{t}))-\Gamma_{\mathrm{k}} \theta_{\mathrm{k}}, \dot{\theta}_{\mathrm{k}}=\mathrm{L}_{\mathrm{g}_{\mathrm{k}}} \mathrm{Q}(\mathrm{x}(\mathrm{t})), \quad \Pi_{\mathrm{k}}>0, \quad \Gamma_{\mathrm{k}} \geq 0, k=1, \cdots m .
$$

The exponential stability under feedforward control (this assumption holds for the turbo-fan engine model) and appropriate, reasonable observability conditions guarantee the closed-loop stability.

The approach of Barrier Lyapunov Functions (BLFs) ${ }^{[7,8,15,16]}$ is based on augmenting barrier functions reflective of the constraints being enforced to the Lyapunov function. If a control law can be designed to guarantee that the augmented Lyapunov function is non-increasing along the trajectories, the constraints cannot be exceeded since the BLF-related terms in the Lyapunov function increase when the state approaches the bounds. The combined BLF and SG approach was successfully used in our prior work on stratified charge direct injection automotive engine control ${ }^{[10,11]}$ to coordinate fueling rate, spark timing, exhaust gas recirculation valve and throttle actuators subject to various pointwise-in-time state and control constraints. This approach is also pursued here.

Following the Barrier Lyapunov Function approach and our previous work ${ }^{[10,11]}$ we propose an augmented goal function, which is a sum of the original goal function augmented by the risk functions:

$$
Q_{a}=Q\left(y_{0}\right)+R_{1}\left(y_{1}\right)+\cdots+R_{l}\left(y_{l}\right) .
$$

With the Speed-Gradient control law derived from $Q_{a}$, the control action is determined by the original goal function when the constrained outputs are far from violating the bounds, and it starts to be driven by the gradient of the appropriate risk function when one of these outputs is close to its bounds. Since the SG controller dynamically minimizes $Q_{a}$, the constrained output is "pushed away" from its bound.

In the application of the SG methodology to the reduced order turbo-fan engine model, most of the constrained outputs and the tracking output in the reduced order model have direct feed-through terms, i.e., they have the form,

$$
y_{i}=h_{i}\left(x, u_{i}\right) .
$$

These direct feed-through terms appear primarily because the dynamics of majority of the engine states is faster that that of core and fan speeds. When model order reduction is performed, the dynamics of fast states are approximated 
by the static relationships that express them in terms of the slow states and inputs thereby leading to direct control feedthrough terms.

To address the presence of these direct feedthrough terms, we can decompose the goal function into a sum of a state dependent term and a state and control dependent term:

$$
Q_{a}(x, u)=Q_{1 a}(x)+Q_{2 a}(x, u)
$$

We then use the following SG control law for the i-th actuator:

$$
u_{i}=u_{d, i}-\Pi_{\mathrm{i}} \mathrm{L}_{\mathrm{g}_{\mathrm{i}}} \mathrm{Q}_{1 \mathrm{a}}+\overline{\mathrm{u}}_{\mathrm{i}}, \dot{\overline{\mathrm{u}}}_{\mathrm{i}}=-\Gamma_{1 \mathrm{i}} \mathrm{L}_{\mathrm{g}_{\mathrm{i}}} \mathrm{Q}_{1 \mathrm{a}}(\mathrm{x}(\mathrm{t}))-\Gamma_{2 \mathrm{i}} \frac{\partial \mathrm{Q}_{2 \mathrm{a}}}{\partial \mathrm{u}_{\mathrm{i}}}(\mathrm{x}(\mathrm{t}), \mathrm{u}(\mathrm{t})), \Pi_{\mathrm{i}} \geq 0, \Gamma_{1 \mathrm{i}} \geq 0, \Gamma_{2 \mathrm{i}} \geq 0 .
$$

This control law is based on extending the relative degree from the goal function to the control signal through the integrator augmentation.

\section{B. Functional Limiters}

The risk functions can be incorporated into the design of model-based functional limiters. For instance, a functional limiter on a control variable $u$, which appears as the direct-feedthrough term for a given output, can be defined to maintain a risk function value below a prescribed value:

$$
R_{i}\left(h_{i}(x, u)\right) \leq \bar{R} \Rightarrow u_{\min , i}(x, \bar{R}) \leq u \leq u_{\max , i}(x, \bar{R})
$$

The disadvantage of using the functional limiter is that an accurate model of the constraint and its inverse are necessary. Developing and tuning such a model of the constraint can require time and effort. Note, however, that for constrained outputs that cannot be directly measured, the development of a constraint model may not be avoidable.

The SG controller does not rely on the model to the same extent as a functional limiter. Specifically, the SG controller relies more on nonlinear feedback of the closeness between a constrained output (which can be measured or estimated by a model) and the bound and delivers a high gain control action when this error is small to push the output away from the constraint. At the same time, this high gain behavior of SG controller can, in principle, cause chattering in the control and output signals when the discrete-time implementation with slow sampling is used.

\section{Reference Governor}

It turns out that several issues appear to preclude the effective use of the SG controller alone to enforce the engine constraints. Firstly, if the SG controller relies on a particular actuator to enforce a certain constraint but this actuator, in the process of enforcing the constraint, runs into limits, the constraint may become violated. Secondly, the analysis of the turbo-fan engine model (see next Section) has revealed that certain combinations of inputs and constrained outputs exhibit nonminimum phase characteristics. This nonminimum phase behavior appears to preclude the use of certain actuators to deliver high gain response to certain output variables when these output variables approach their bounds.

To address the above difficulties, a prediction of the potential for future constraint violation and an advance action which adjusts the set-point command to the SG controller are beneficial. The set-point adjustment can be performed by the reference governor ${ }^{[5,6]}$. See Figure 4 . The reference governor modifies the set-point, $r(t)$, to which the nominal controller responds to the virtual set-point, $v(t)$, which is then passed to the controller. The reference governor is a first order, discrete-time linear filter with an adjustable bandwidth parameter $\kappa(t)$ :

$$
v(t+1)=v(t)+\kappa(t)(r(t)-v(t)), 0 \leq \kappa(t) \leq 1
$$




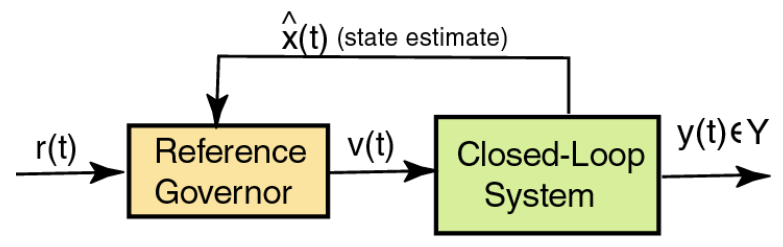

Figure 4. The reference governor for the set-point modification.

The value of $\kappa(t)$ is maximized subject to the constraint that the predicted closed-loop response does not violate the specified constraints in terms of the risk function exceeding a prescribed value. The prediction is performed by applying the usual state transition formula to a discrete-time linear model. The model uncertainty in the prediction can be accounted by developing and incorporating disturbance representation which accounts for this uncertainty ${ }^{[6]}$

\section{Analysis and control design using linearized engine model}

The goal function was selected as

$$
Q(x)=\frac{\gamma_{1}}{2}\left(E P R-E P R_{d}\right)^{2}+\frac{\gamma_{2}}{2}\left(V S V-V S V_{d}\right)^{2}+\frac{\gamma_{3}}{2}\left(V B V-V B V_{d}\right)^{2},
$$

where $E P R$ denotes the engine pressure ratio, VSV denotes the Variable Stator Vane position and $V B V$ denotes the Variable Bleed Valve position. The set-points are denoted by $(\cdot)_{d}$, and $\gamma_{i}, i=1,2,3$, are the weights. This goal function is augmented with the risk functions for constraints on engine output variables. The outputs of the model include core and fan speeds $\left(N_{c}\right.$ and $N_{f}$, respectively), exhaust temperature $\left(T_{50}\right)$, combustor inlet pressure $\left(P s_{3}\right)$, engine thrust $\left(F_{n}\right)$, Low Pressure Compressor Surge/Stall margin $(L P M)$, High Pressure Compressor Surge/Stall margin $(H P M)$, etc. In addition to $V S V$ and $V B V$, fueling rate $\left(W_{f}\right)$ is also the control signal.

The linearized model was derived from the nonlinear NASA C-MAPSS40k high fidelity engine model ${ }^{[13]}$ at different trim points, and the properties of this linearized model were examined. The following observations were made. The dynamics of the linearized system are second order with two states, $N_{c}$ and $N_{f}$. The steady-state equilibria of these dynamics are asymptotically stable with two real negative eigenvalues. Most of the outputs have the direct feed-through terms. These direct feed-through terms are created in the model principally as a result of model order reduction through elimination of the fast states.

It is also of interest to examine the zeros in different input and output channels. It can be shown that the system is nonminimum phase from either $W_{f}$ or $V S V$ as an input to $L P M$ as output; however, it is minimum phase from $V B V$ as an input to $L P M$ as an output. Physically, the difference in the behavior is due to both $W_{f}$ and $V S V$ being "downstream" actuators for the Low Pressure Compressor, while VBV represents an "upstream" actuator. Consistently with this observation on the zeros, our initial application of the Speed Gradient Control has revealed the difficulty in enforcing the $L P M$ constraint using either $W_{f}$ or $V S V$ as inputs. The $V B V$ actuator appears to therefore be most suitable to deal with the $L P M$ constraint. Given the saturation limits on $V B V$, the reference governor can be helpful in modifying the set-point for $E P R, E P R_{d}$, to enforce the constraints. It may also be desirable to adjust the steady-state set-point schedule for $V B V$, i.e., $V B V_{d}$, to provide sufficient bi-directional authority to $V B V$ to enforce the $L P M$ constraints.

Similar analysis was conducted for other input-output pairs that together with simulation assessments has helped to finalize the controller architectural decisions. Two potential implementations are summarized in Tables 1 and 2.

The differences between the two implementations are in the use of the SG controller versus the functional limiters on $W_{f}$ to enforce $T_{50}$ and $H P M$ constraints. The implementation based on the functional limiters to enforce these two constraints (Table 1) computes the maximum admissible quantity of fuel that can be injected without causing violation of these two constraints, and then the fuel quantity is limited to this maximum admissible quantity. The implementation based on the SG controller (Table 2) relies on feedback on deviation of $T_{50}$ and HPM from the 
bounds to adjust the time rate of change of $W_{f}$. The second implementation is more consistent with our overall design approach; it provides responses that are essentially equivalent, but, as our simulation results indicate, it may cause slight chattering in the fuel quantity due to discrete-time implementation if the update rate is sufficiently low. This chattering occurs when the engine rides $T_{50}$ and HPM constraints while responding to the thrust increase command. The effects of chattering can be lessened by suitably choosing the form of the risk functions, tuning parameters of the risk functions, or by increasing the sampling frequency if permissible by the microcontroller characteristics.

Compared to the SG controller and to the functional limiters, the implementation of a predictive reference governor is more involved in terms of computing time and effort. To alleviate the computational burden in our implementation, the reference governor is applied to handling only certain constraints (on LPM, as previously discussed, and also on $W_{f} / P_{s 3}$ ). Furthermore, it is assumed that only the fuel input changes over the prediction horizon while $V B V$ and $V S V$ remain fixed. Note that during a runway incursion avoidance maneuver, the emphasis is on engine acceleration and total thrust provided. Thus, alternative, simpler implementations may be possible where the reference governor is replaced by a fixed gain filter.

\begin{tabular}{|c|c|c|c|}
\hline Output & $\begin{array}{c}\text { Main control actuator in } \\
\text { Speed-Gradient control } \\
\text { law }\end{array}$ & Functional Limiter on & Reference Governor on \\
\hline$E P R$ set-point tracking & $W_{f}$ & & \\
\hline$N_{c}$ constraint & $W_{f}$ & & \\
\hline$N_{f}$ constraint & $W_{f}$ & & $E P R$ set-point \\
\hline$L P M$ constraint & $V B V$ & & \\
\hline$H P M$ constraint & $V S V, V B V$ & maximum of $W_{f}$ & \\
\hline$T_{50}$ constraint & $V S V, \mathrm{VBV}$ & maximum of $W_{f}$ & \\
\hline$W_{f /} P s_{3}$ constraint & & & $E P$ set-point \\
\hline
\end{tabular}

Table 1. Controller architectural decisions for the implementation which uses functional limiters on $W_{f}$ to handle constraints on $T_{50}$ and $H P M$.

\begin{tabular}{|c|c|c|c|}
\hline Output & $\begin{array}{c}\text { Main actuator in Speed- } \\
\text { Gradient control law }\end{array}$ & Functional Limiter on & Reference Governor on \\
\hline$E P R$ set-point tracking & $W_{f}$ & & \\
\hline$N_{c}$ constraint & $W_{f}$ & & \\
\hline$N_{f}$ constraint & $W_{f}$ & & \\
\hline$L P M$ constraint & $V B V$ & & \\
\hline$H P M$ constraint & $V S V, V B V$, & & \\
& $W_{f}$ & & $E P R$ set-point \\
\hline$T_{50}$ constraint & $V S V, \mathrm{VBV}$, & & \\
\hline$W_{f /} P s_{3}$ constraint & & & \\
\hline
\end{tabular}

Table 2. Controller architectural decisions for the implementation which uses Speed-Gradient control to handle constraints on $T_{50}$ and $H P M$.

Simulation results from an aggressive engine performance scenario that showcases the controller capability to respond to fast thrust commands and dynamically changing constraints are shown in Figure 5. After a period of initialization when the model states settle down to a low thrust condition, at time $10 \mathrm{sec}$, a full engine thrust is commanded. Stringent constraints are imposed on all the output variables, in particular, on surge margins. The engine thrust reaches the steady-state thrust level within $4 \mathrm{sec}$. The constraints are made even more stringent at time $25 \mathrm{sec}$ when the command to decrease the thrust is given to the engine. The reference governor becomes active and slows down the drop in the EPR set-point while $V B V$ opens up to mitigate the $L P M$ constraint. After $25 \mathrm{sec}$, the constraint on fan speed becomes progressively stricter with time, emulating a potential and progressive fault 
condition. Another command to increase the thrust is given at time $40 \mathrm{sec}$ but now under significantly tighter constraints. The full thrust level cannot be reached, nevertheless the controller behaves in a stable and predictable manner while enforcing stringent constraints. The set-points are followed whenever feasible and tracking performance is gracefully degraded under more stringent constraints. The VSV and VBV actuators assist the fuel actuation in mitigating $T_{50}$ constraint during thrust increase phases, without causing $L P M$ constraint violation.

For comparison, the implementation in Table 2 was also tested. The responses are similar, see Figure 6 . The main difference is the appearance of light chattering in $T_{50}$ and $H P M$ signals when the functional limiters for $T_{50}$ and HPM are replaced by SG control law and the discrete-time implementation of the controller is used. In the simulation, the temperature limit was decreased by 50 deg to cause $T_{50}$ to ride the constraint longer at $10 \mathrm{sec}$ and demonstrate the chattering effect. The chattering in fuel as $T_{50}$ and HPM ride the constraints is negligible and has no apparent effect on thrust.

Finally, the controller configured to track set-points in core speed, $N_{c}$, instead of set-points in EPR has also been successfully tested in simulations. This controller version was not pursued further and the controller for tracking set-points in $E P R$ was retained.
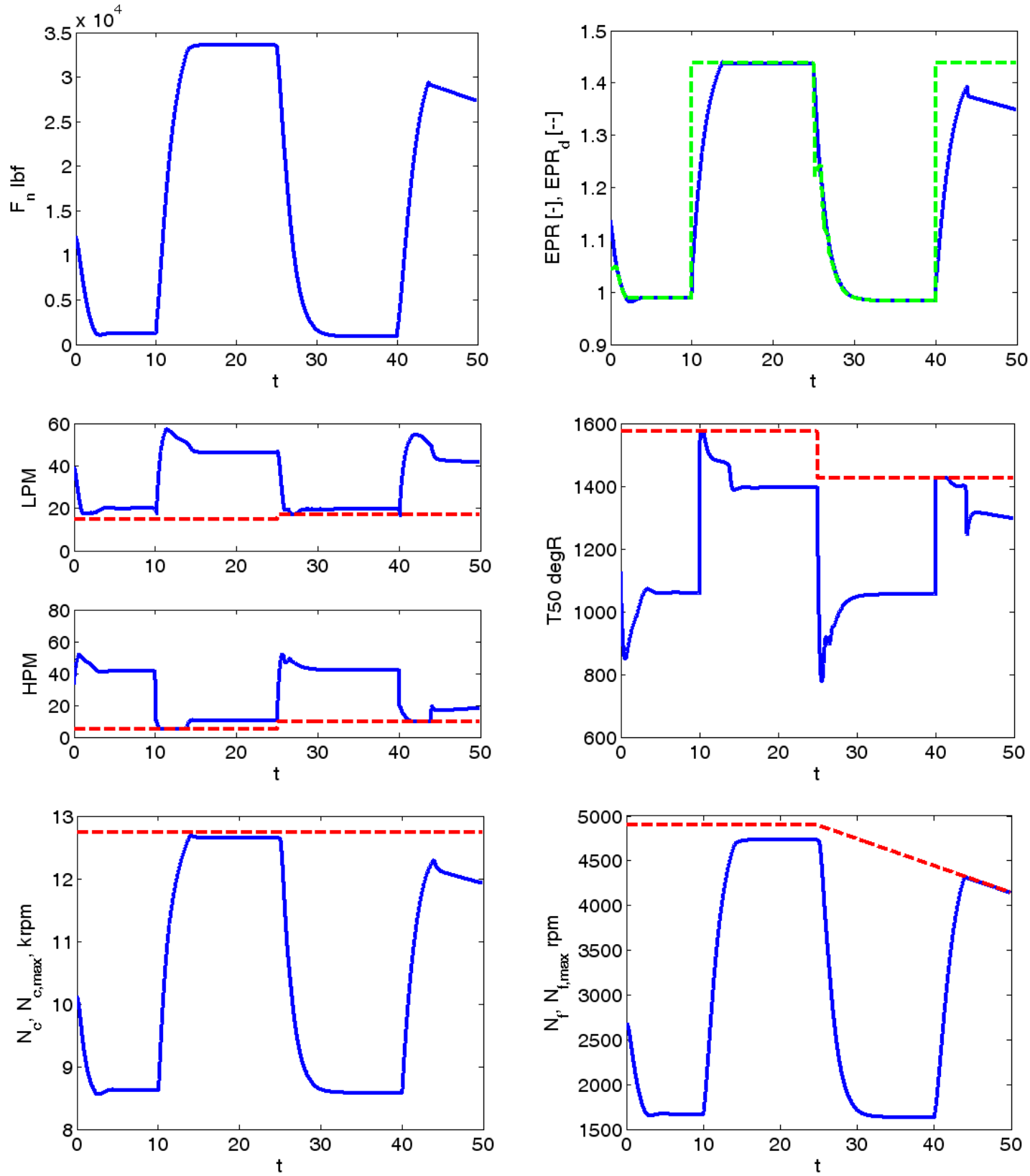

9

American Institute of Aeronautics and Astronautics 

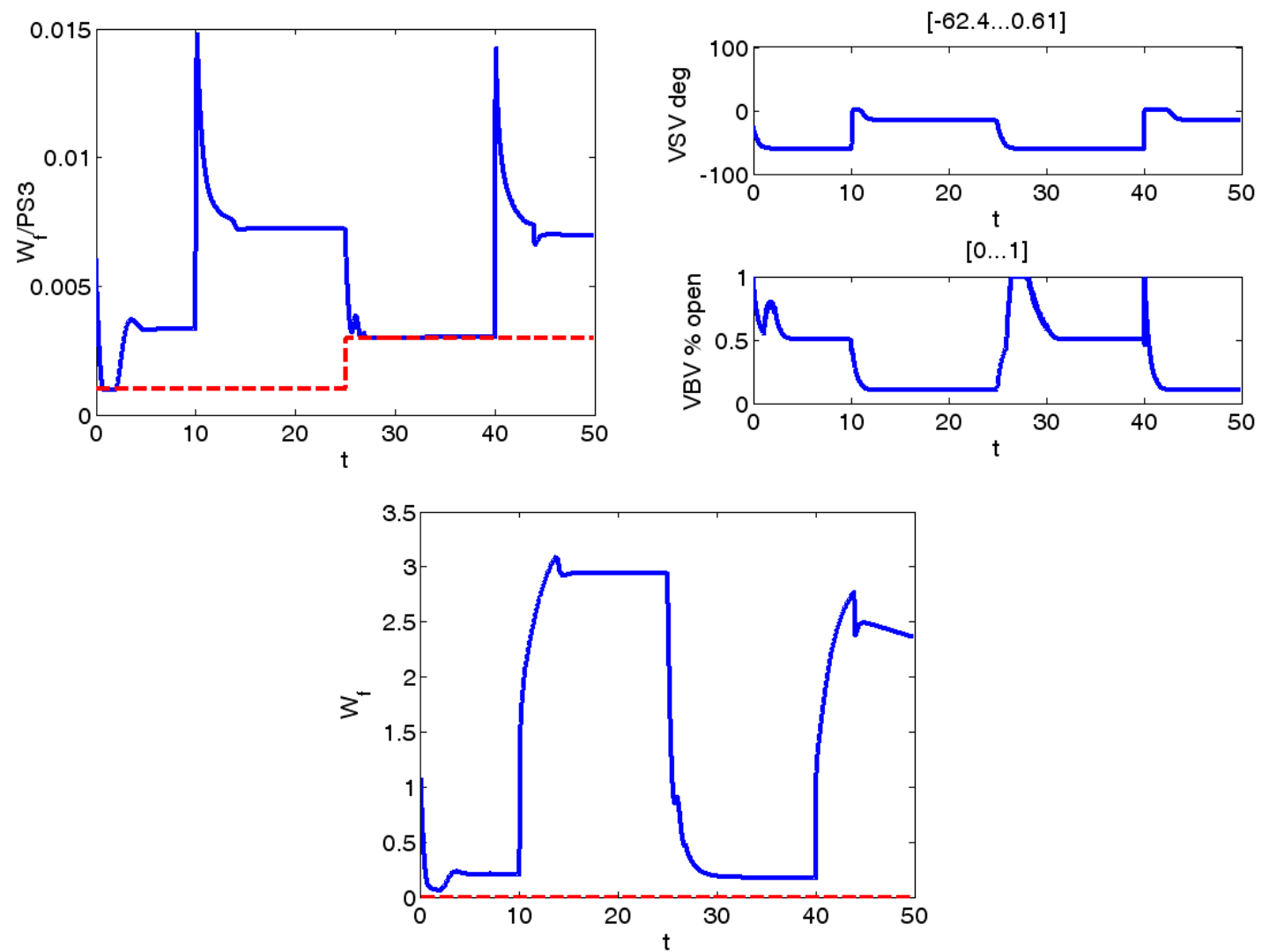

Figure 5. Engine response based on the linearized model simulations. Time histories of engine variables (solid lines) and limits/set-points (dashed lines).
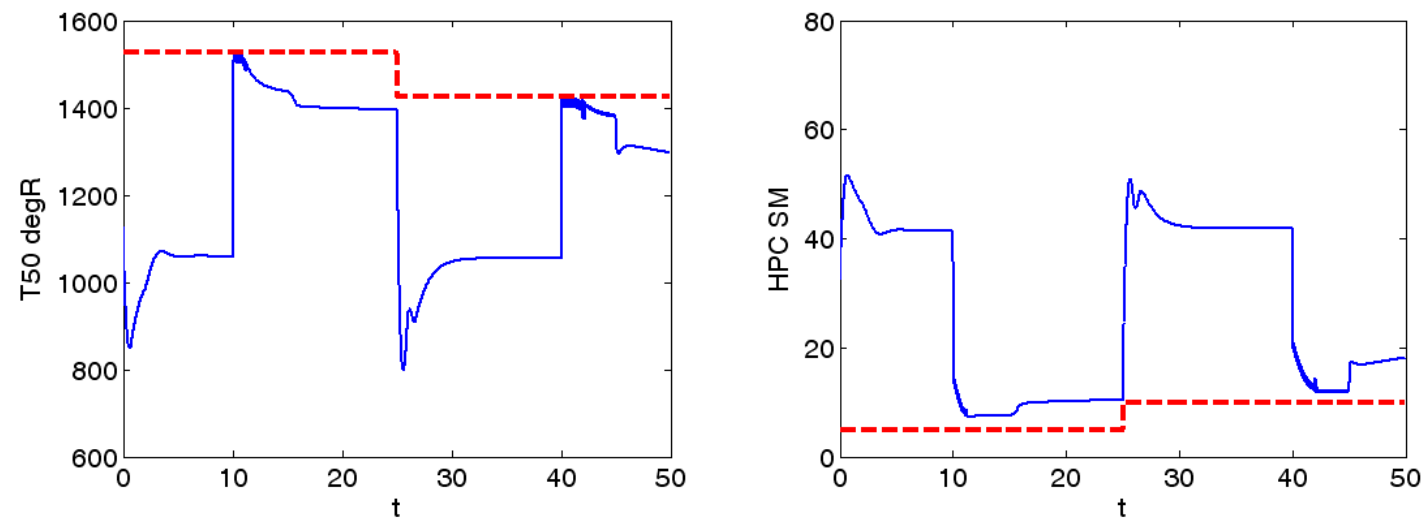

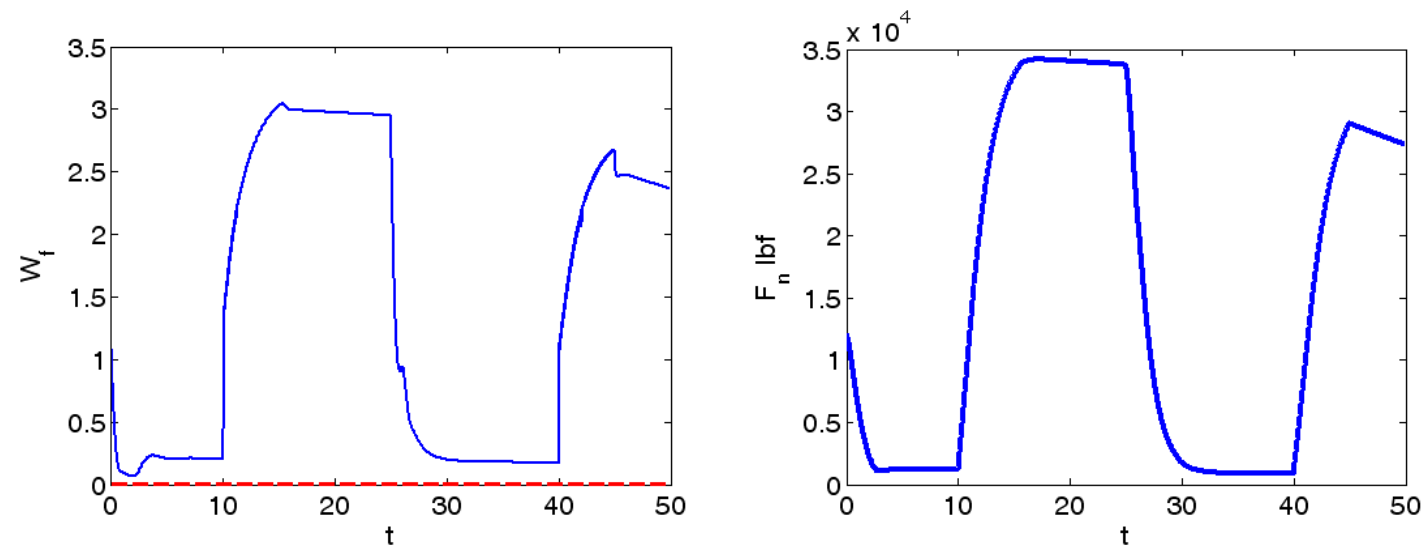

Figure 6. Engine response based on the linearized model simulations. Time histories of engine variables (solid lines) and limits/set-points (dashed lines). Slight chattering emerges in $\boldsymbol{T}_{\mathbf{5 0}}, \boldsymbol{H P M}$ and $W_{f}$ signals when the functional limiters for $T_{50}$ and $H P M$ constrained outputs are replaced by $S G$ control law.

\section{Simulation results on high fidelity C-MAPSS40k engine model}

The controller has been implemented on a high fidelity C-MAPSS40k engine model. In addition to being nonlinear, the high fidelity turbo-fan engine model exhibits higher order dynamics and fast modes that are not present in the reduced order linearized model. The high fidelity model also includes previously unmodelled actuator dynamics. Consequently, the overall retuning and re-calibration of the controller was performed.

Figure 6 shows a runway incursion-related simulation scenario where the increase in the engine thrust is commanded at time $5 \mathrm{sec}$ and is further increased at time $6 \mathrm{sec}$ to respond to the emergency maneuvering request.

The constraints on $T_{50}$ and HPM are relaxed at time $6 \mathrm{sec}$ to facilitate the emergency maneuver. The responses of the developed controller are compared with the response of the nominal controller in the C-MAPSS40 k engine model, responding to the same thrust increase command. The developed controller provides an improvement over the existing controller in terms of increased thrust response.
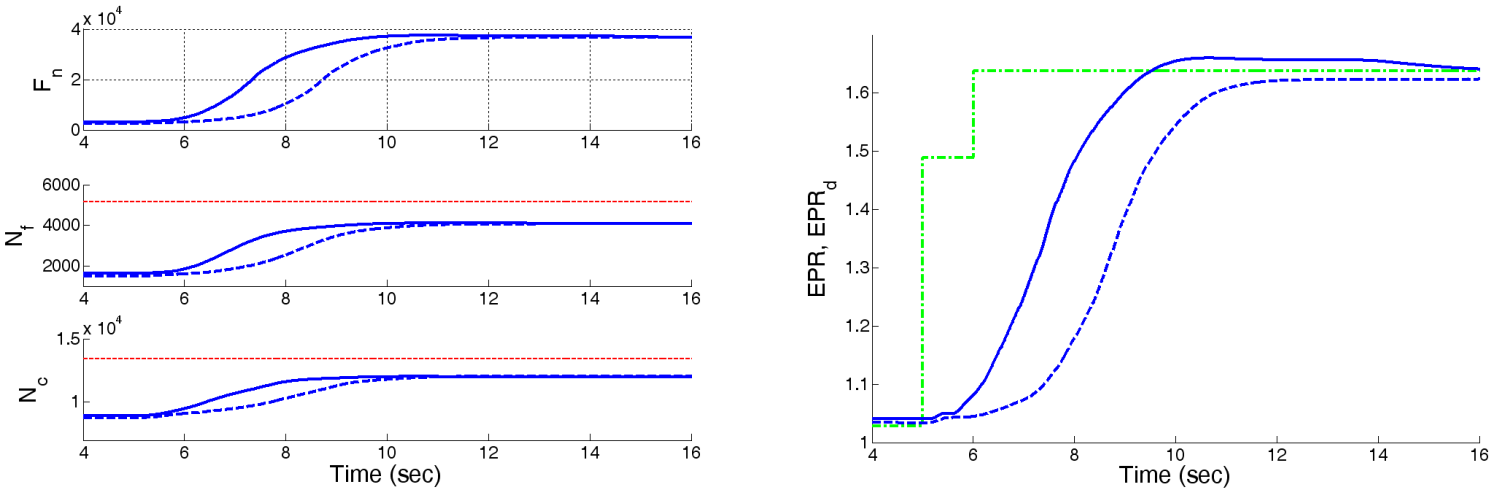

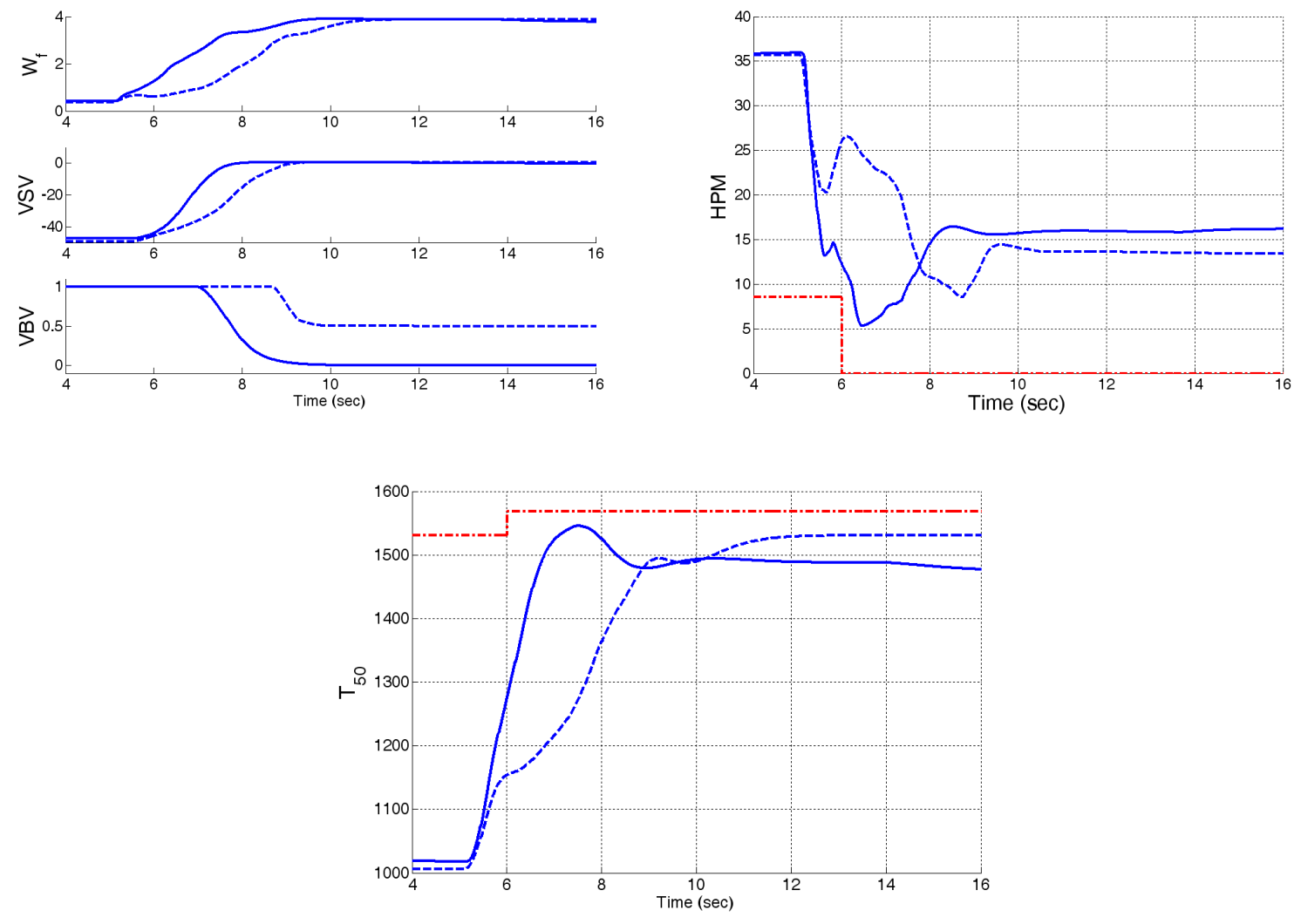

Figure 6. Time histories of the engine variable with the developed controller (solid) versus the nominal CMAPSS40k controller (dashed). The set-points/limits are indicated by dash-dotted lines.

\section{Conclusions}

Risk functions can be defined based on individual engine condition monitoring to intelligently relax the constraints during aircraft emergency maneuvering and provide faster and higher overall levels of thrust based on the individual engine condition monitoring. In this paper, approaches to incorporate risk functions in the control design for the turbo-fan engine are identified. The primary approach is based on application of the Speed-Gradient control framework. In it, risk functions are used to augment the goal function which is dynamically minimized with the Speed-Gradient control law. Consequently, the risk functions determine the control action near the constraint boundaries and enforce the constraints. The advantage of the Speed-Gradient control is that it is computationally low complexity and that it relies on feedback to handle constraints. Types of constraints that can be handled with this approach have been delineated in the paper. A reference governor, which predictively maintains risk functions below threshold values, can be augmented to handle the types of constraints that the Speed-Gradient control law may not handle effectively, while the functional limiters configured to maintain risk functions below prescribed values may be introduced to avoid chattering for certain constraints that emerges at lower sampling rates.

Simulation results, based on both linearized and high fidelity engine models, have confirmed the objectives of this research. Namely, that it is possible to provide faster and higher overall levels of engine thrust in response to emergency conditions. Additionally, we have shown a design approach which can flexibily handle a multitude of time varying constraints by coordinating multiple actuators. The design approach incorporates the notion of risk to intelligently relax constraints to achieve performance goals. Future work may include an investigation to further generalize the approach to enable limit based controls to be designed for a wide variety of applications. Additionally, engine controls designed via this approach may be combined within a hierarchical control to demonstrate its utility in an integrated engine and flight control for emergency situations. 


\section{Acknowledgments}

This paper describes work supported by NASA (Award Number NNC08CA54C) on Fast Response Engine Research (FasTER).

\section{References}

${ }^{1}$ Burcham, F.W., Maine, T.A., Burken, J., and Bull, J., 'Using Engine Thrust for Emergency Flight Control: MD-11 and B747 Results,” Technical Memorandum, NASA/TM-1998-206552, NASA, 1998.

${ }^{2}$ Csank, J., May, R.D., Litt, J.S., and Guo, T.- H., "Control Design for a Generic Commercial Aircraft Engine," $46^{\text {th }}$ AIAA/ASME/SAE/ASEE Joint Propulsion Conference and Exhibit, 25-28 July 2010, Nashville, TN, 2010, AIAA $2010-6629$.

${ }^{3}$ DeCastro, J.A., Litt, J.S., and Frederick, D.-K., "A Modular Aero-Propulsion System simulation of a Large Commerical Aircraft Engine," NASA/TM-2008-215303, AIAA 2008-4579.

${ }^{4}$ Fradkov, A.L., Adaptive Control in Complex Systems, Nauka, 1990.

${ }^{5}$ Gilbert, E.G., Kolmanovsky, I., and Tan, K.-T., "'Discrete-time Reference Governors and the Nonlinear Control of Systems with State and Control Constraints," International Journal of Robust and Nonlinear Control, Vol. 5, No. 5, pp. 487-504, 1995.

${ }^{6}$ Gilbert, E.G., and Kolmanovsky, I., "Fast Reference Governors for Systems with State and Control Constraints and Disturbance Inputs,” International Journal of Robust and Nonlinear Control, Vol. 9, No. 15, pp. 1117-1141, 1999.

${ }^{7}$ Guldner, J., and Utkin, V.I., 'Sliding Mode Control for Gradient Tracking and Robot Navigation using Artificial Potential Fields," IEEE Transactions on Robotics and Automation, Vol. 16, No. 5, pp. 615-620.

${ }^{8}$ Hermann, G., Turner, M.C., and Postlethwaite, I., "A Robust Override Scheme Enforcing Strict Output Constraints for a Class of Strictly Proper Systems,', Automatica, Vol. 44, pp. 753-760, 2008.

${ }^{9}$ Jaw, L.C., and Mattingly, J.D., Aircraft Engine Controls: Design, System Analysis, and Health Monitoring,AIAA Education Series, 2009.

${ }^{10}$ Kolmanovsky, I., and Yanakiev, D., "Speed Gradient Control of Nonlinear Systems and its Applications to Automotive Engine Control," Journal of Society of Instrumentation and Control Engineers (SICE), Vol. 47, No. 3, 2008, pp. 160-169.

${ }^{11}$ Kolmanovsky, I., Druzhinina, M., and Sun, J., "Speed Gradient Approach to Torque and Air-to-Fuel Ratio Control in DISC Engines," IEEE Transactions on Control Systems Technology, Vol. 10, No. 5, 2002.

${ }^{12}$ Litt, J.S., Frederick, D.K., and Guo, T.-H., "The Case for Intelligent Propulsion Control for Fast Engine Response," AIAA Infotech@Aerospace Conference, 6-9 April, 2009, Seattle, Washington, AIAA paper 2009-1876.

${ }^{13}$ May, R. D., Csank, J., Lavelle, T.M., Litt, J.S., and Guo, T.- H., “A High Fidelity Simulation of a Generic Commercial Aircraft Engine and Controller," 46 ${ }^{\text {th }}$ AIAA/ASME/SAE/ASEE Joint Propulsion Conference and Exhibit, 25-28 July 2010, Nashville, TN, 2010, AIAA 2010-6630.

${ }^{14}$ Merrill, W., “A Risk Model for Engine Controller Design,” Technical Report, SMI Report \# NNC08CA54C-TR-01_Rev_0.

${ }^{15}$ Sane, H., and Bernstein, D.S., “Robust Nonlinear Control of the Electromagnetically Controlled Oscillator,'” Proceedings of the American Control Conference, Anchorage, AK, May 8-10, 2002, pp. 809-814.

${ }^{16}$ Tee, K.P., Ge, S.S., and Tay, E.H. "Barrier Lyapunov Functions for the Control of Output Constrained Systems," Automatica, Vol. 45, No. 4, pp. 918-927, 2009.

${ }^{17}$ Thompson, A., Hacker, J., and Cao, C., "Adaptive Engine Control in the Presence of Output Limits," Proceedings of AIAA Infotech@Aerospace, Atlanta, Georgia, 2010. 